\title{
Histone deacetylase 6 reduction promotes aortic valve calcification via an endoplasmic reticulum stress-mediated osteogenic pathway
}

Zurong Fu, MD, ${ }^{a}$ Fei Li, MD, ${ }^{b}$ Liangliang Jia, MD, PhD, ${ }^{a}$ Shengan Su, MD, ${ }^{a}$ Yaping Wang, MD, Zhejun Cai, $\mathrm{MD},{ }^{\mathrm{a}, \mathrm{c}}$ and Meixiang Xiang, $\mathrm{MD}, \mathrm{PhD}^{\mathrm{a}}$

\section{ABSTRACT}

Objective: Aortic valve (AoV) calcification occurs via a pathophysiologic process that includes osteoblastic differentiation of valvular interstitial cells (VICs). Histone deacetylases (HDACs) have been shown to be involved in the pathogenesis of vascular diseases. Here, we investigated the role of HDAC6 in AoV calcification.

Methods: AoV cusps from patients with aortic stenosis $(\mathrm{n}=7)$ and normal controls $(\mathrm{n}=7)$ were subjected to determination of calcified nodules and HDAC6 expression. Human VICs were cultured in osteogenic media and treated with $10 \mathrm{uM}$ tubacin or HDAC6 small interfering RNA silencing to inhibit HDAC6. Treatment with $100 \mathrm{uM}$ tauroursodeoxycholic acid was used to suppress endoplasmic reticulum stress. Activating transcription factor 4 (ATF4) small interfering RNA was used to knock down ATF4. Alizarin red staining was used to evaluate calcified nodules formation of VICs cultured with osteogenic media for 14 days.

Results: HDAC6 expression was significantly reduced in AoV tissue of patients with aortic stenosis compared with controls. Tubacin treatment or HDAC6 silencing markedly promoted osteoblastic differentiation accompanied by endoplasmic reticulum stress activation in VICs. The HDAC6 inhibition-induced osteogenic pathway was mediated by endoplasmic reticulum stress/ATF4 pathway as indicated by tauroursodeoxycholic acid pretreatment or ATF4 silencing. Finally, alizarin red staining showed that HDAC6 inhibition promoted osteoblastic differentiation of VICs, which could be suppressed by tauroursodeoxycholic acid.

Conclusions: HDAC6 inhibition promotes AoV calcification via an endoplasmic reticulum stress/ATF4-mediated osteogenic pathway. HDAC6 may be a novel target for AoV calcification prevention and treatment. ( $\mathrm{J}$ Thorac Cardiovasc Surg 2019;158:408-17)

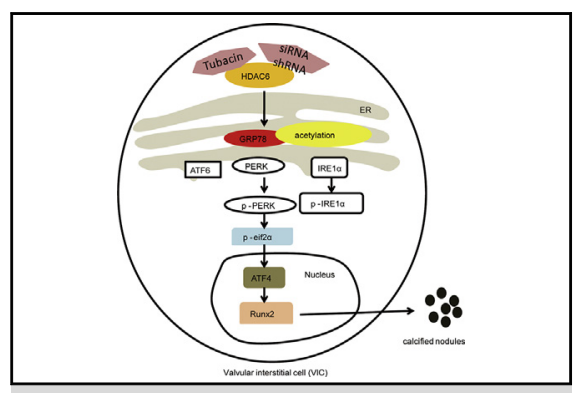

HDAC6 suppression promotes aortic valve calcification via ER stress.

\section{Central Message}

HDAC6 reduction is linked with AoV calcification. Inhibition of HDAC6 promotes osteoblastic differentiation of VICs via ER stress.

\section{Perspective}

HDACs have been involved in the pathogenesis of cardiovascular diseases, but their roles in AoV calcification remain unknown. Here we show HDAC6 reduction in the AoV cusps of patients with CAVD. Mechanistically, HDAC6 suppression promotes osteoblastic differentiation of VICs via an ER stress/ ATF4-mediated osteogenic pathway.

See Commentary page 418 .
Calcified aortic valve disease (CAVD) that may finally develop into aortic valve (AoV) stenosis is the most common valvular disease in developed countries and is a

From the ${ }^{a}$ Department of Cardiology, The Second Affiliated Hospital, Zhejiang University School of Medicine, Key Lab of Cardiovascular Disease of Zhejiang Province, Hangzhou, Zhejiang, China; ${ }^{\mathrm{b}}$ Department of Cardiovascular Surgery, Union Hospital, Tongii Medical College, Huazhong University of Science and Technology, Wuhan, Hubei, China; and ${ }^{\mathrm{c}}$ The Key Laboratory of Cardiovascular Remodeling and Function Research, Chinese Ministry of Education, Chinese National Health Commission and Chinese Academy of Medical Sciences, The State and Shandong Province Joint Key Laboratory of Translational Cardiovascular Medicine, Department of Cardiology, Qilu Hospital of Shandong University, Jinan, China.

Supported by grants from Provincial and Ministry Joint Major Projects of National Health Commission of China (WKJ-ZJ-1703 to M.X.), and the National Natural Science Foundation of China (No. 81670259 to M.X., No. 81670390 to Z.C., and No. 81670351 to F.L.).

Drs Fu and Li contributed equally to this article.

Received for publication May 10, 2018; revisions received Oct 9, 2018; accepted for publication Oct 12, 2018; available ahead of print Dec 19, 2018. significant cause of morbidity and mortality. ${ }^{1}$ CAVD is characterized by mineralized nodules formation on the valve cusps and is not a passive degenerative process but

Address for reprints: Meixiang Xiang, MD, PhD, Department of Cardiology, The Second Affiliated Hospital, Zhejiang University School of Medicine, Key Lab of Cardiovascular Disease of Zhejiang Province, 88 Jiefang Rd, Hangzhou, Zhejiang 310009, China (E-mail: xiangmx@zju.edu.cn); or Zhejun Cai, MD, Department of Cardiology, The Second Affiliated Hospital, Zhejiang University School of Medicine, Key Lab of Cardiovascular Disease of Zhejiang Province, 88 Jiefang Rd, Hangzhou, Zhejiang 310009, China. The Key Laboratory of Cardiovascular Remodeling and Function Research, Chinese Ministry of Education, Chinese National Health Commission and Chinese Academy of Medical Sciences, The State and Shandong Province Joint Key Laboratory of Translational Cardiovascular Medicine, Department of Cardiology, Qilu Hospital of Shandong University, Jinan, China (E-mail: caizhejun@zju.edu.cn). $0022-5223 / \$ 36.00$

Copyright (C) 2018 by The American Association for Thoracic Surgery https://doi.org/10.1016/j.jtcvs.2018.10.136 

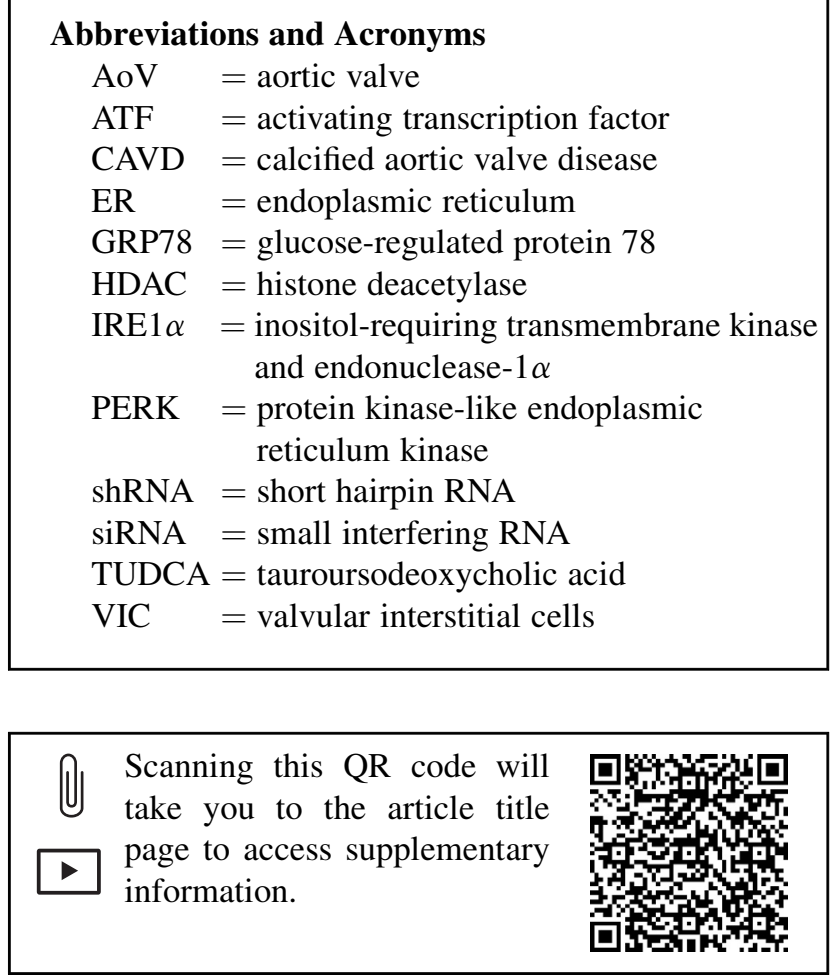

rather a chronic active disease process, including lipoprotein deposition, valve fibrosis, and osteoblastic differentiation of valvular interstitial cells (VICs). ${ }^{2}$ However, there is no effective pharmacologic therapy other than surgical or interventional AoV replacement. ${ }^{3-5}$

Histone deacetylases (HDACs) are a group of enzymes that lead to the deacetylation of the lysine residues on histone and nonhistone proteins. ${ }^{6}$ It has been reported that HDAC inhibitors promote osteoblast maturation. ${ }^{7}$ Azechi and colleagues ${ }^{8}$ found that inhibition of HDAC activity by nonspecific inhibitor trichostatin A can promote vascular calcification. Kwon and colleagues ${ }^{9}$ found that HDAC1 has a protective effect on vascular calcification. These studies indicate that HDAC may also be involved in the pathogenesis of AoV calcification.

The endoplasmic reticulum (ER) is a crucial luminal network for protein synthesis, folding, and transportation. ${ }^{10}$ ER homeostasis disruption leads to accumulation of unfolded and misfolded proteins in the ER lumen and creates a state defined as ER stress, which activates a complex signaling network termed the unfolded protein response. Unfolded protein response consists of 3 branches: protein kinase-like ER kinase (PERK), inositol-requiring transmembrane kinase and endonuclease- $1 \alpha$ (IRE $1 \alpha$ ), and activating transcription factor (ATF) $6 .{ }^{11}$ ER stress is associated with various pathophysiologic states, ${ }^{12-15}$ including AoV calcification. ${ }^{10,16,17}$ It has also been reported that ER stress, especially the
PERK/ATF4 pathway, is highly involved in the development of AoV calcification. ${ }^{10,18}$

HDAC6 is a member of the HDAC IIb subfamily. ${ }^{19}$ Rao and colleagues ${ }^{11}$ found that inhibition of HDAC6 in breast cancer cells induces acetylation of glucose-regulated protein 78 (GRP78) and triggers ER stress. ${ }^{11}$ Because ER stress is involved in AoV calcification, we therefore hypothesized that HDAC6 reduction may promote AoV calcification by activating the ER stress-mediated osteogenic pathway.

\section{MATERIALS AND METHODS \\ Human AoV Samples}

The human study was approved by an ethics committee of Union Hospital, Tongji Medical College, Huazhong University of Science and Technology. Human specimens were from the Department of Cardiovascular Surgery, Union Hospital, Tongji Medical College, Huazhong University of Science and Technology. According to the Declaration of Helsinki, informed consents were signed. From October 2017 to April 2018, calcified and normal AoV were collected. We obtained calcified AoVs from patients who underwent valve replacement due to symptomatic, severe aortic stenosis without moderate or severe aortic regurgitation or mitral valve diseases $(n=7)$. Normal AoVs were from patients with acute Stanford type A aortic dissection accompanied by moderate aortic insufficiency (more than Grade II) who underwent emergency Bentall surgery $(n=7)$. Patients with bicuspid aortic valves and connective tissue disorders were excluded.

The tissues were put in phosphate buffered saline on ice after harvest and then were transported to the lab immediately. The tissues were divided into 3 parts. One part was used to extract protein for Western blot, 1 part was fixed in $4 \%$ paraformaldehyde for immunohistochemical staining and Von Kossa staining, and 1 part was digested by collagenase for cell culture and treatment.

\section{Cell Culture and Treatment}

VICs were isolated from normal AoV cusps by collagenase digestion. ${ }^{16}$ All experiments were performed with cells in passage 3 to 5 . After $70 \%$ confluence, cells were incubated with indicated interventions in Dulbecco's modification of Eagle medium supplemented with 5\% fetal bovine serum, $10 \mathrm{mM} \beta$-glycerophosphate (Sigma-Aldrich, St Louis, Mo), $10 \mathrm{nM}$ dexamethasone (Sigma-Aldrich), $4 \mathrm{ug} / \mathrm{mL}$ cholecalciferol (Sigma-Aldrich), and $8 \mathrm{mM}$ calcium chloride (Sigma-Aldrich). If needed, pharmacologic reagents, including $10 \mu \mathrm{M}$ tubacin (Selleckchem, Houston, Tex), $100 \mu \mathrm{M}$ tauroursodeoxycholic acid (TUDCA) (Calbiochem, San Diego, Calif) were added.

\section{Cell Transfection of Small Interfering RNAs}

Small interfering RNAs (SiRNAs) against indicated proteins were provided by Santa Cruz Biotechnology (Santa Cruz, Calif) and were designed against human HDAC6 or human ATF4. Scrambled siRNAs (Santa Cruz Biotechnology) were used as normal control. VICs were transfected with scrambled or indicated siRNAs using RNAiMAX (Invitrogen, Waltham, Mass) and Opti-MEM (Gibco, Carlsbad, Calif) according to manufacturer's recommendations.

\section{Lentivirus Transfection}

VICs were infected with recombinant lentiviruses expressing control short hairpin (shRNA) or HDAC6 shRNA (Jikai Genechem Co Ltd, Shanghai, China) according to manufacturer's instruction.

\section{Western Blot}

Tissue samples and cells were homogenized and subsequently performed for Western blotting as previously described. ${ }^{20}$ The following 
antibodies were used: phosphorylated IRE1 $\alpha$ (1:1000 dilution) (RB2150924) from Thermo Fisher Scientific (Waltham, Mass) phosphorylated PERK (1:500 dilution) (sc-32577) and $\beta$-actin (1:1000 dilution) (sc-47778) from Santa Cruz Biotechnology, Runx2 (1:1000 dilution) (8486S), phosphorylated eukaryotic initiation factor $2 \alpha$ (1:1000 dilution) (9721S) from Cell Signaling Technology (Danvers, Mass), and HDAC6 (1:1000 dilution) (12834-1-AP) from Proteintech (Rosemont, Ill). $\beta$-actin was used as normalization for total protein.

\section{Histopathology and Immunohistochemistry}

Human AoV tissues were rapidly harvested, rinsed in phosphate buffered saline, fixed in $4 \%$ paraformaldehyde in phosphate buffered saline, and embedded in paraffin for histopathologic analyses. Immunohistochemical staining was performed using the following antibodies: HDAC6 (1:200 dilution) (12834-1-AP) from Proteintech. At least 2 of the $3 \mathrm{AoV}$ cusps for each patient were analyzed.

\section{Von Kossa Staining}

Sections were stained with a von Kossa staining kit (Abcam, Cambridge, United Kingdom) according to manufacturer's instruction. ${ }^{17}$

\section{Alizarin Red Staining \\ For analysis of osteoblastic differentiation in VICs, cells were incubated with indicated interventions in a conditioning media (growth medium supplemented with $10 \mathrm{mM} \beta$-glycerophosphate, $10 \mathrm{nM}$ dexamethasone, $4 \mu \mathrm{g} / \mathrm{mL}$ cholecalciferol, and $8 \mathrm{mM}$ calcium chloride) for 14 days as reported previously. ${ }^{17,21}$ Media were changed every 3 days. Alizarin red staining for calcium deposits was performed as described. ${ }^{22}$ To quantitatively analyze alizarin red stain, wells were rinsed with distilled water, and alizarin red stains were bleached with $10 \%$ acetic acid at $85^{\circ}$ C. Supernatant was spectrophotometrically analyzed at $450 \mathrm{~nm} .^{22}$ \\ Statistical Analysis \\ All values are presented as mean \pm standard deviation. After confirming that all variables were normally distributed using the Kolmogorov-Smirnov test and visualized by Q-Q plot, statistical differences were evaluated by Student $t$ test or analysis of variance followed by Bonferroni's multiple comparison test by SPSS version 18.0 (IBM-SPSS Inc, Armonk, NY).}

\section{Sample Size Estimation}

Stata software version 12.0 (StataCorp, College Station, Tex) was used to estimate the sample size for 2-sample comparison of mean in human study. Sample size was set as identical in 2 groups. Alpha was set as 0.05 , power was set as 0.8 . Results of mean and standard deviation values in the human study were used.

\section{RESULTS \\ HDAC6 Is Reduced in AoV Cusps of Patients With CAVD}

To confirm whether HDAC6 is involved in AoV calcification, we first determined the levels of HDAC6 in AoV samples from patients with CAVD and normal controls. The clinical characteristics of patients are listed in Table 1. Von Kossa staining confirmed a clear calcium deposit in the AoV tissues from patients with CAVD (Figure 1, A). Immunohistochemical staining (Figure 1, $B$ ) and Western blot (Figure 1,C) revealed that HDAC6 was significantly reduced in calcified AoV samples compared with controls.
TABLE 1. Clinical characteristics in patients with calcified aortic valve disease (CAVD) versus control patients

\begin{tabular}{|c|c|c|c|}
\hline Characteristic & $\begin{array}{l}\text { Control } \\
(n=7)\end{array}$ & $\begin{array}{l}\text { CAVD } \\
(n=7)\end{array}$ & $\begin{array}{c}P \\
\text { value }\end{array}$ \\
\hline Age (y) & $62.6 \pm 5.2$ & $64.8 \pm 6.1$ & .523 \\
\hline Male gender & $4(57.1)$ & $5(71.4)$ & .577 \\
\hline Body mass index & $22.9 \pm 3.1$ & $23.2 \pm 5.1$ & .908 \\
\hline Smoking & $5(71.4)$ & $6(85.7)$ & .515 \\
\hline Hypertension & $3(42.9)$ & $5(71.4)$ & .28 \\
\hline Hypercholesterolemia & $4(57.1)$ & $6(85.7)$ & .237 \\
\hline Diabetes mellitus & $2(28.6)$ & $3(42.9)$ & .577 \\
\hline Coronary heart disease & $2(28.6)$ & $3(42.9)$ & .577 \\
\hline Peripheral vascular diseases & $0(0)$ & $1(14.3)$ & .299 \\
\hline Aortic insufficiency* & $7(100)$ & $0(0)$ & $<.001$ \\
\hline Antiplatelets & $3(42.9)$ & $6(85.7)$ & .094 \\
\hline ACEi/ARB & $2(28.6)$ & $3(42.9)$ & .577 \\
\hline Beta blockers & $1(14.3)$ & $0(0)$ & .299 \\
\hline Calcium channel blockers & $1(14.3)$ & $0(0)$ & .299 \\
\hline Diuretics & $0(0)$ & $2(28.6)$ & .127 \\
\hline Statins & $3(42.9)$ & $6(85.7)$ & .094 \\
\hline LVEF $(\%)$ & $55.3 \pm 3.5$ & $52.1 \pm 7.3$ & .363 \\
\hline Transvalvular gradient $(\mathrm{mm} \mathrm{Hg})$ & $15.4 \pm 5.5$ & $79.7 \pm 6.3$ & $<.001$ \\
\hline Aortic valve area $\left(\mathrm{cm}^{2}\right)$ & $3.1 \pm 0.4$ & $0.7 \pm 0.1$ & $<.001$ \\
\hline
\end{tabular}

Values are presented as mean \pm standard deviation or $\mathrm{n}(\%)$. $C A V D$, Calcified aortic valve disease; $A C E i$, angiotensin-converting enzyme inhibitor; $A R B$, angiotensin II receptor blockers; $L V E F$, left ventricular ejection fraction. *Defined as Grade I or greater.

\section{Inhibition of HDAC6 Activates an Osteogenic Pathway in VICs}

VICs are the main cellular components of AoVs, and the activation of osteogenic pathways is an important mechanism of AoV calcification. ${ }^{2,10}$ VICs were incubated with tubacin, a specific inhibitor of HDAC6. As indicated in Figure 2, $A$, and Figure E1, tubacin treatment markedly increased the expression of Runx2 in VICs. The effect of HDAC6 on Runx2 was further confirmed by HDAC6 silencing in VICs because knockdown of HDAC6 significantly induced Runx 2 expression (Figure 2, B).

\section{HDAC6 Reduction Induces ER Stress in VICs}

A previous report indicates that HDAC6 suppression can activate ER stress in breast cancer cells. ${ }^{11}$ We determined ER stress under HDAC6 inhibition in VICs. As shown in Figure 3, A, and Figures E1 and E2, HDAC6 inhibition by tubacin significantly triggered the expressions of ER stress markers, except for ATF6, in VICs. Moreover, knockdown of HDAC6 by siRNA in VICs showed the same result as tubacin treatment; that is, HDAC6 silencing markedly induced ER stress in VICs (Figure 3, B). 

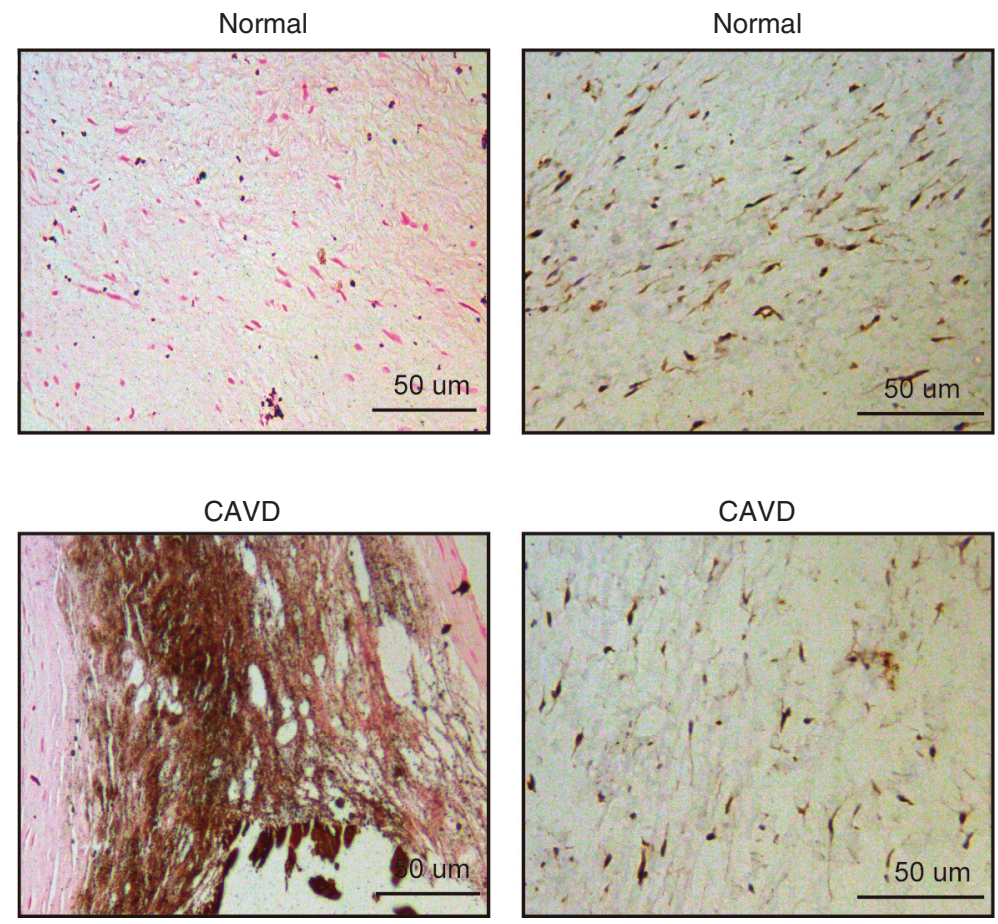

A

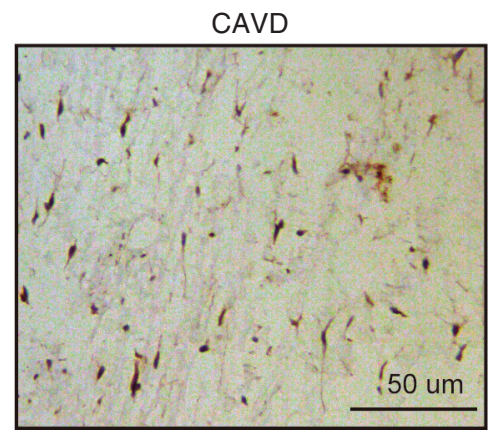

B
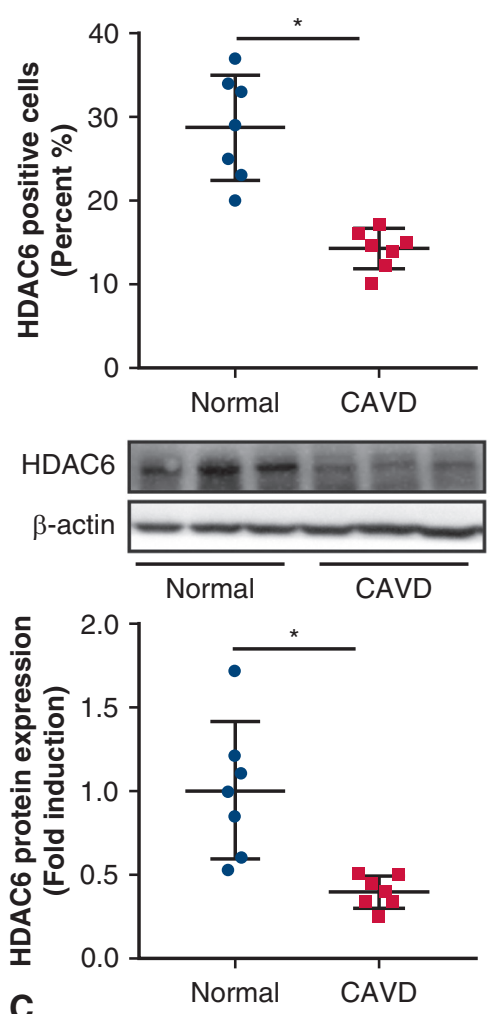

c

FIGURE 1. Histone deacetylase 6 (HDAC6) is reduced in aortic valve cusps of patients with calcified aortic valve disease (CAVD). A, Representative von Kossa staining of the AoV cusps of normal controls and patients with CAVD. The AoV cusps of patients with CAVD showed significant calcified nodules formation. B, Immunohistochemical staining of HDAC6 in the AoV cusps of normal controls and patients with CAVD. HDAC6 staining was significantly reduced in AoV cusps of patients with CAVD. C, Western blot analysis of HDAC6 in AoV cusps from normal controls and patients with CAVD. HDAC6 expression was significantly reduced in AoV cusps of patients with CAVD. Scale bar: $50 \mu \mathrm{m} . \mathrm{N}=7$ for each group. $* P<.05$ versus normal.
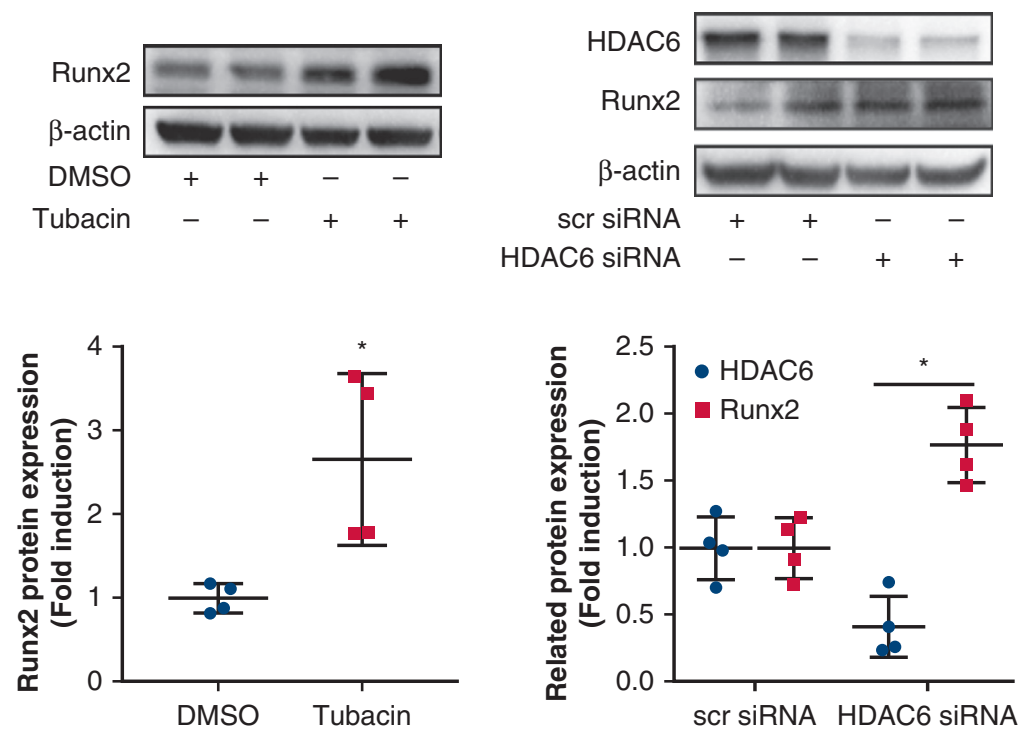

A

B

FIGURE 2. Inhibition of histone deacetylase 6 (HDAC6) activates an osteogenic pathway in valvular interstitial cells (VIC). A, Tubacin (10 uM) treatment significantly increased Runx2 expression in VIC. B, Knockdown of HDAC6 significantly induced Runx2 expression in VIC. *P $<.05$ versus dimethyl sulfoxide (DMSO) or scrambled small interfering RNA (scr siRNA). Runx2, Runt-related transcription factor 2. 

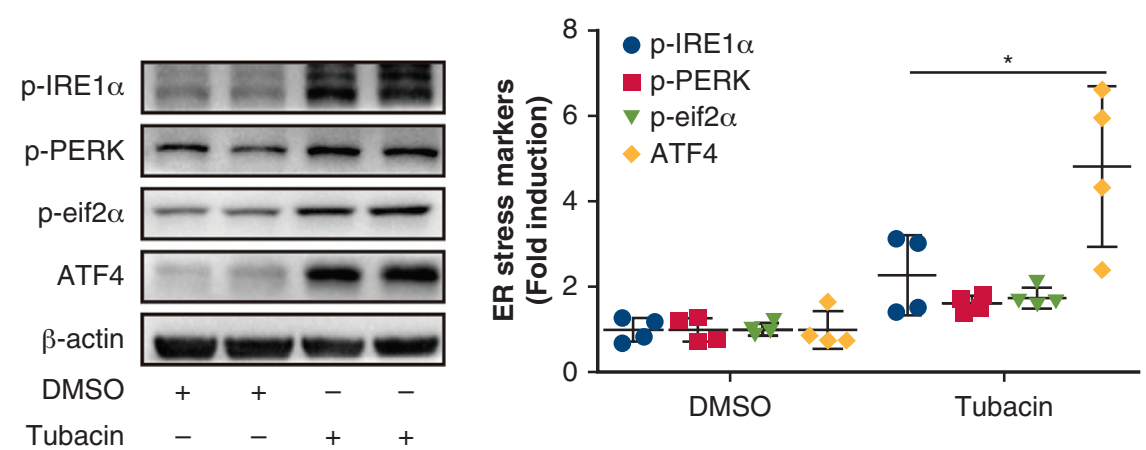

A
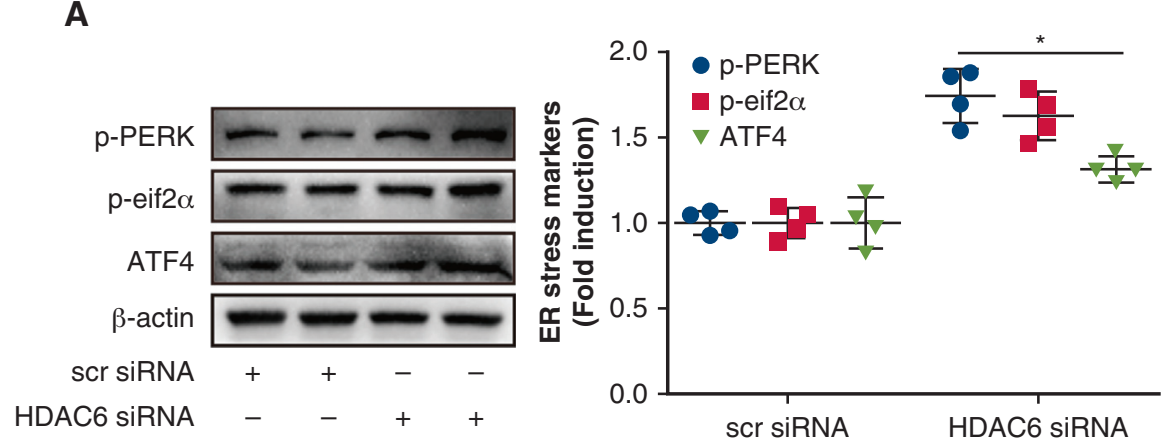

B

FIGURE 3. Histone deacetylase 6 (HDAC6) reduction induces endoplasmic reticulum (ER) stress in valvular interstitial cells (VIC). A, Tubacin (10 uM) treatment significantly increased the expressions of ER stress markers phosphorylated protein kinase-like ER kinase $(p-P E R K)$, phosphorylated inositol-requiring transmembrane kinase and endonuclease- $1 \alpha$ (p-IRE1 $\alpha$ ), phosphorylated eukaryotic initiation factor $2 \alpha$ ( $p$-eif $2 \alpha$ ), and activating transcription factor 4 (ATF4) in VIC. B, HDAC6 silencing markedly induced the expression of ER stress markers (p-PERK, p-eif2 $\alpha$, and ATF4) in VIC. $* P<.05$ versus dimethyl sulfoxide $(D M S O)$ or scrambled small interfering RNA (scr siRNA).

\section{The Effect of HDAC6 Inhibition on ER Stress and Osteogenic Pathway Activation Is More Profound in VICs From Normal Control Patients}

We checked the effect of HDAC6 inhibition in VICs from normal AoVs and from calcified AoVs. As shown in Figure E3, both ER stress markers phosphorylated PERK and ATF4, and osteogenic pathway markers Runx2 and alkaline phosphatase were significantly induced in VICs from calcified AoVs. HDAC6 inhibition by tubacin led to a more significant induction of ER stress and osteogenic pathways in VICs from the control AoVs (Figure E3).

\section{HDAC6 Suppression-Induced Osteogenic Pathway Is Mediated by ER Stress in VICs}

Our previous reports confirm that ER stress activation induces AoV calcification. ${ }^{10,16,17}$ Because our data show that HDAC6 inhibition triggers ER stress and osteogenic pathways in VICs, the next question is whether alleviating ER stress rescues osteogenic pathway activation induced by HDAC6 suppression. As depicted in Figure 4, $A$ and $B$, preincubation of TUDCA, the ER stress suppressor, ${ }^{10}$ significantly relieved ER stress activation as well as Runx 2 expression after tubacin treatment. We also tested the effect of TUDCA in HDAC6-silenced cells. Inhibition of ER stress by TUDCA markedly reduced Runx2 expression that was induced by HDAC6 silencing (Figure 4, $C$ and $D$ ).

\section{ATF4 Activation Mediates Osteogenic Pathway Activation Triggered by HDAC6 Inhibition}

ER stress promotes oxidized low-density lipoproteininduced osteoblastic differentiation of VICs through the ATF4 pathway. ${ }^{10}$ We tested whether an HDAC6 suppression-induced osteogenic pathway is mediated by ATF4. Knockdown of ATF4 significantly suppressed Runx 2 expression that was induced by tubacin treatment (Figure 5, A-C) or HDAC6 silencing (Figure 5, D-F) in VICs.

\section{Alleviating ER Stress Prevents Osteoblast Differentiation of VICs Induced by HDAC6 Inhibition}

The activation of the osteogenic pathway leads to the osteoblastic differentiation of VICs, resulting in intracellular and extracellular calcium deposition and the formation of calcified nodules. ${ }^{2}$ The final question would be whether osteoblast differentiation induced by HDAC6 suppression could be alleviated by ER stress inhibition. VICs were transfected with lentiviral HDAC6 shRNA or scramble shRNA. The transfected cells were incubated 

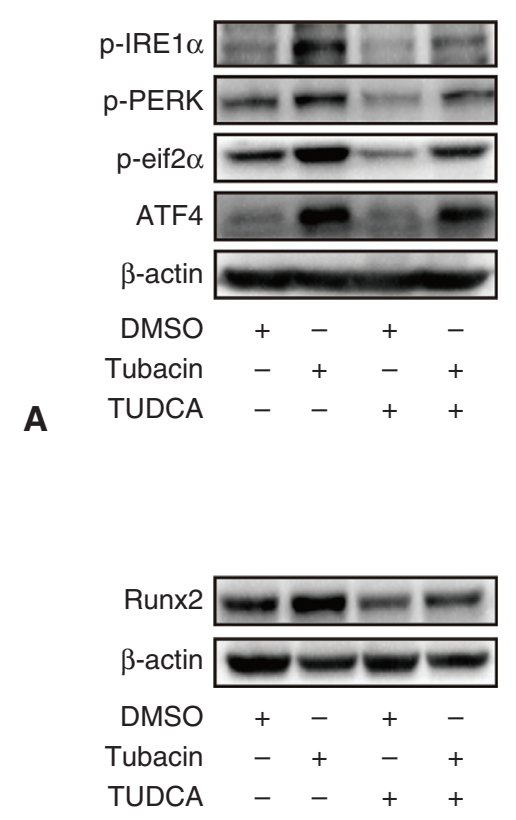

B

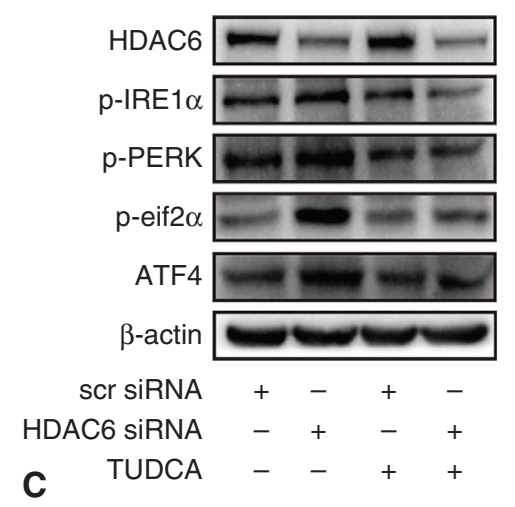

\section{D}

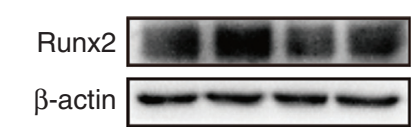

$\begin{array}{rllll}\text { scr siRNA } & + & - & + & - \\ \text { HDAC6 siRNA } & - & + & - & + \\ \text { TUDCA } & - & - & + & +\end{array}$
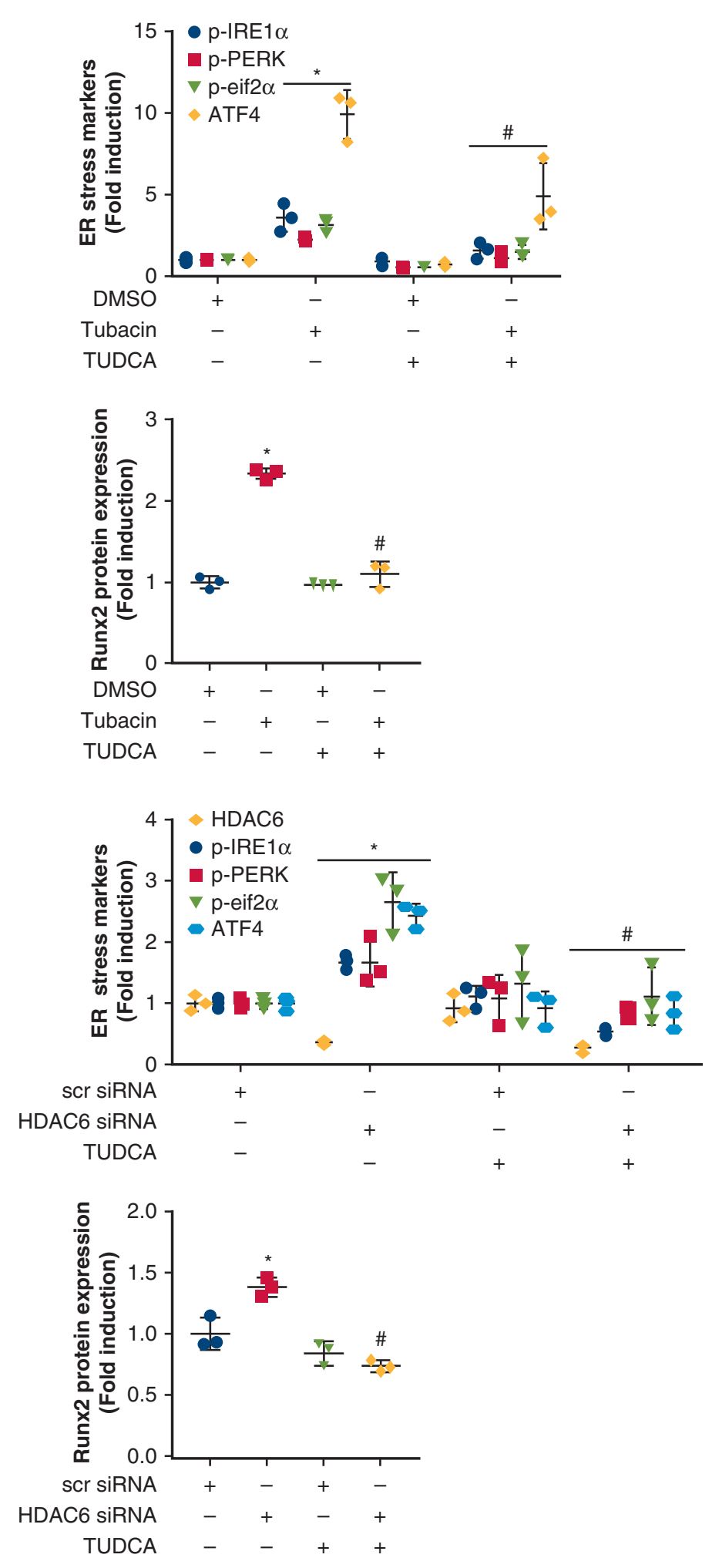

FIGURE 4. Histone deacetylase 6 (HDAC6) suppression-induced osteogenic pathway is mediated by endoplasmic reticulum (ER) stress in valvular interstitial cells (VIC). A, Tauroursodeoxycholic acid (TUDCA) (100 uM) preincubation significantly relieved ER stress activation as indicated by expressions of phosphorylated protein kinase-like ER kinase ( $p$-PERK), phosphorylated inositol-requiring transmembrane kinase and endonuclease-1 $\alpha$ $(p-I R E 1 \alpha)$, phosphorylated eukaryotic initiation factor $2 \alpha$ (p-eif $2 \alpha$ ), and activating transcription factor 4 (ATF4) induced by tubacin (10 uM). B, TUDCA pretreatment markedly reduced Runx2 expression induced by tubacin. C, TUDCA incubation significantly attenuated ER stress activation (p-PERK, p-IRE1 $\alpha$, p-eif $2 \alpha$, and ATF4) caused by HDAC6 silencing. D, TUDCA treatment markedly suppressed Runx 2 expression induced by HDAC6 silencing. ${ }^{*} P<.05$ versus dimethyl sulfoxide $(D M S O)$ or scrambled small interfering RNA (scr siRNA). \#P<.05 versus tubacin or HDAC6 siRNA. Runx2, Runtrelated transcription factor 2 . 


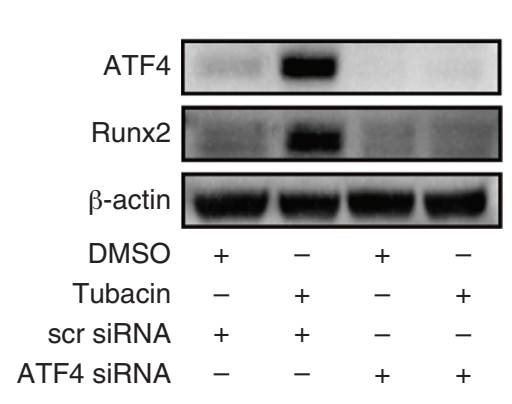

A

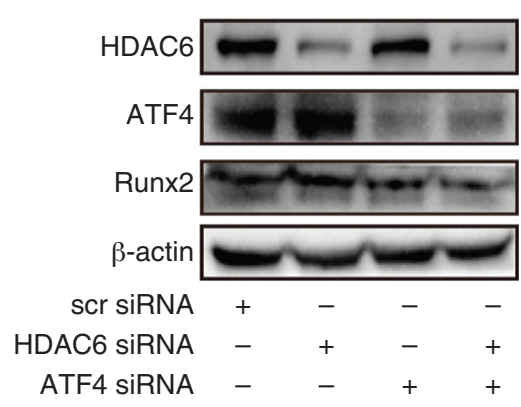

D

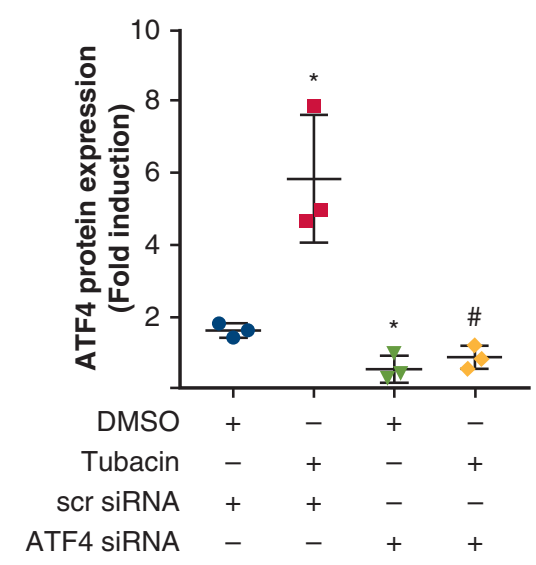

B

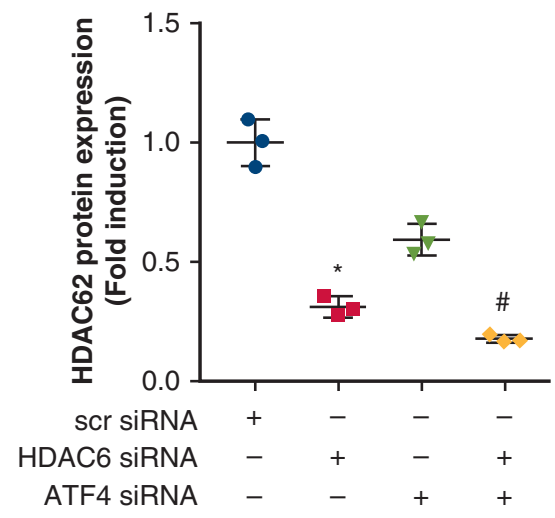

E

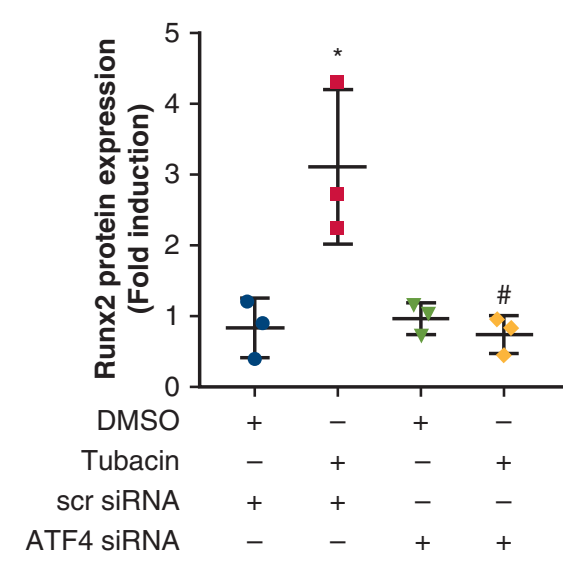

C

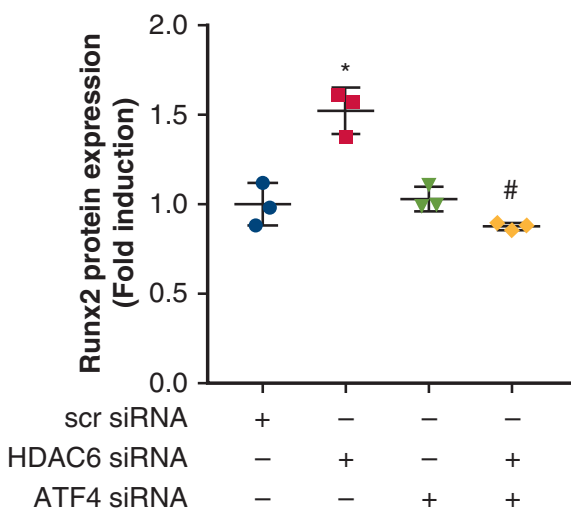

$\mathbf{F}$

FIGURE 5. Activating transcription factor 4 (ATF4) mediates osteogenic pathway activation triggered by histone deacetylase 6 inhibition (HDAC6). A to C, Knockdown of ATF4 significantly suppressed Runx 2 expression induced by tubacin treatment in valvular interstitial cells (VIC). D to F, Knockdown of ATF4 significantly suppressed Runx2 expression caused by HDAC6 silencing in VIC. $* P<.05$ versus dimethyl sulfoxide (DMSO) or scrambled small interfering RNA (scr siRNA). \#P<.05 versus tubacin or HDAC6 siRNA. Runx2, Runt-related transcription factor 2.

with or without TUDCA and were cultured in osteogenic media for 14 days. As shown in Figure 6, $A$ and $B$, lentiviral HDAC6 shRNA transfection markedly suppressed HDAC6 expression. Alizarin red staining showed that knockdown of HDAC6 promoted calcified nodules formation, whereas inhibition of ER stress by TUDCA suppressed the effect (Figure 6, C).

\section{DISCUSSION}

Despite the clinical importance of heart disease and intense study in AoV calcification, the mechanisms underlying the development of AoV calcification remain unclear. $^{3-5}$ The current study shows that HDAC6 reduction is linked with CAVD in human beings. Our data indicate that HDAC6 inhibition promotes AoV calcification by ER stress/ATF4-mediated osteogenic pathway.

The function of HDACs is transcriptional regulation and posttranslational modification of proteins. ${ }^{6}$ In the field of cardiovascular medicine, studies have found that class I and class II HDAC inhibitors can prevent myocardial hypertrophy and myocardial injury after myocardial infarction, and may inhibit fibrosis. ${ }^{19,23}$ HDAC2 2 and HDAC3 can maintain endothelium integrity and reduce atherosclerosis. ${ }^{19,23,24}$

However, HDAC inhibition seems to be harmful in vascular calcification. Studies have shown that HDAC inhibitors promote vascular calcification. ${ }^{8}$ HDAC1 has a protective effect on vascular calcification, but the study shows that HDAC6 is unchanged in vascular calcification. ${ }^{9}$ Our results show that HDAC6 is reduced in calcified AoV, indicating the pathogenesis of $\mathrm{AoV}$ calcification is not identical to vascular calcification.

Our results demonstrate that HDAC6 inhibition activates ER stress in VICs. The result is in consistent with the studies in cancer cells. ${ }^{11,25} \mathrm{Li}$ and colleagues ${ }^{11}$ reported that HDAC6 prevents acetylation of GRP78, the molecular chaperone that colonizes the $\mathrm{ER},{ }^{11}$ at lysine residue $633{ }^{25}$ In nonstressed cells, GRP78 binds to ER transmembrane receptors and keeps them inactive. When acetylated, GRP78 reduces binding to PERK and IRE1 $\alpha$ on the ER membrane, and activates ER stress. ${ }^{11}$ 


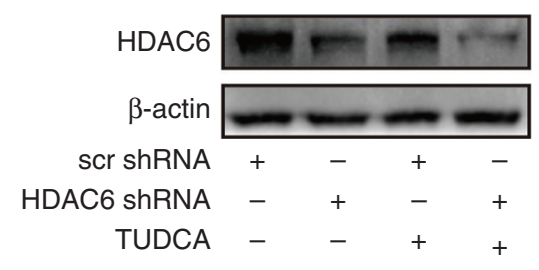

A
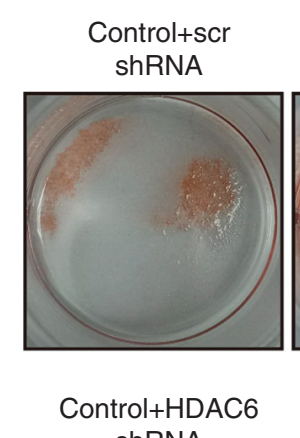
ShRNA

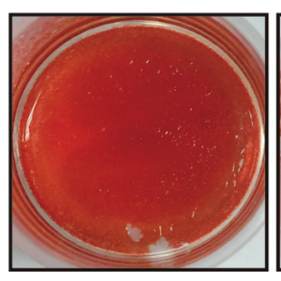

TUDCA+scr shRNA

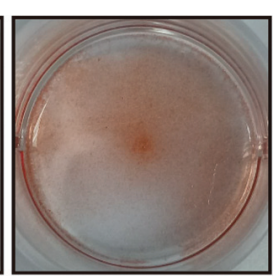

TUDCA+HDAC6 ShRNA

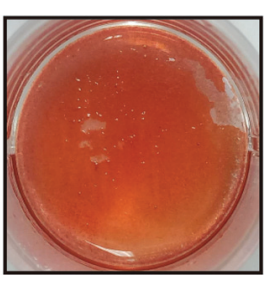

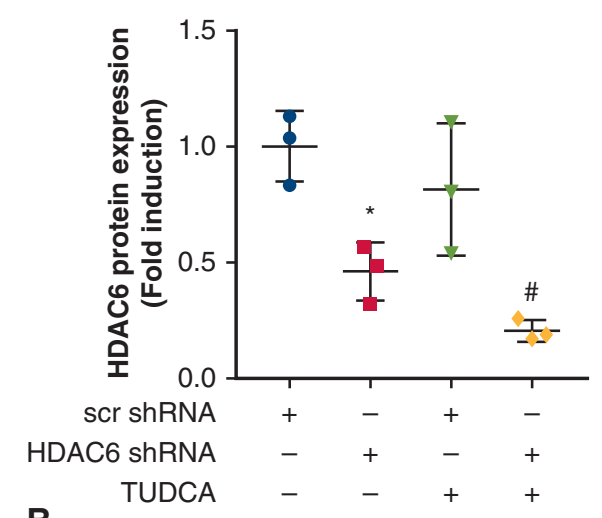

B

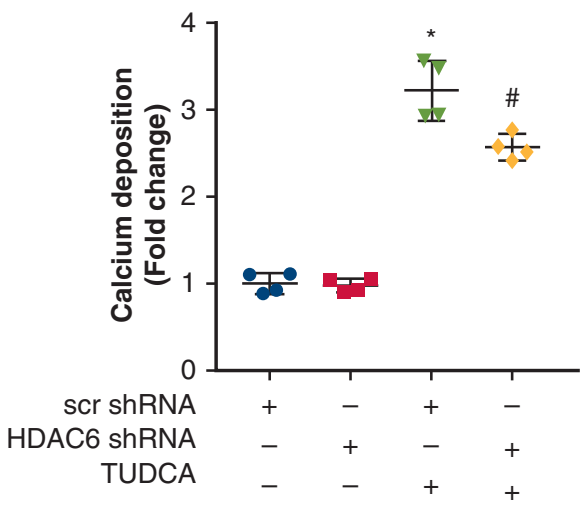

C

FIGURE 6. Alleviating endoplasmic reticulum stress prevents osteoblastic differentiation of valvular interstitial cells (VIC) induced by histone deacetylate 6 (HDAC6) inhibition. A and B, Lentiviral HDAC6 short hairpin RNA (shRNA) transfection markedly suppressed HDAC6 expression in VIC. C, Alizarin red staining showed that knockdown of HDAC6 promoted calcified nodules formation, whereas tauroursodeoxycholic acid (TUDCA) suppressed the effect. $* P<.05$ versus scrambled $(s c r)$ shRNA. $\# P<.05$ versus TUDCA + scr shRNA in panel B or HDAC6 shRNA in panel C.

ATF4 downstream of ER stress serves not only an ER stress sensor but a transcriptional factor of genes involved in osteogenic pathways as well. 18,26,27 Our previous work $^{10,17}$ indicates that ATF4 is essential in the activation of osteoblastic differentiation of VICs. Masuda and colleagues $^{18}$ further confirmed that ATF4 knockout prevents AoV and vascular calcification in vivo. ${ }^{18}$ Because HDAC6 suppression activates ER stress/ATF4 pathway, it is reasonable that ATF4 mediates the osteogenic effect of HDAC6 inhibition in VICs. Indeed, our data show that ATF4 silencing attenuates osteogenic pathway activation induced by HDAC6 suppression.

Runx2 is a transcription factor that is required for osteoblast differentiation. It has been confirmed that Runx2 binds with osteoblast-specific cis-acting element, termed osteoblast-specific cis-acting element, to activate downstream osteoblast-related genes. ${ }^{28}$ Previous studies report Runx2 plays a critical role in smooth muscle cell osteogenic phenotype change and mineral deposition. ${ }^{29}$ Our previous report also shows that Runx 2 is essential in the development of AoV calcification. ${ }^{10}$

Our study shows that HDAC6 inhibition promotes AoV calcification, suggesting that activation of HDAC6 may prevent AoV calcification. However, inhibition of HDAC6 inhibits cardiac fibrosis, ${ }^{30}$ and reduces reactive oxygen species generation and confers a protective effect against myocardial ischemia/reperfusion or hypoxia/reoxygenation injury. ${ }^{31}$ Inhibition of HDAC6 also suppresses endothelial cell migration and angiogenesis, ${ }^{32}$ and prevents endothelial apoptosis. ${ }^{33}$ These data indicate the differential role of HDAC6 in various cell types.

HDACs, including HDAC6 inhibitors, have been applied in clinical trials for their anticancer effects. ${ }^{19,23}$ As our study implicates, HDAC6 inhibition is associated with 


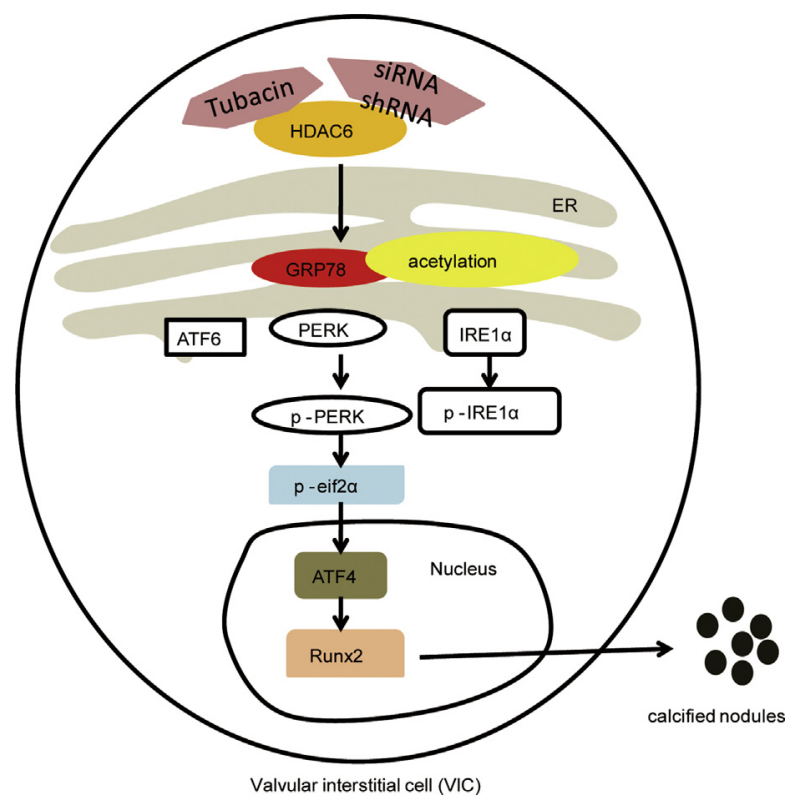

FIGURE 7. Proposed mechanism of histone deacetylase 6 (HDAC6) suppression-triggered osteoblastic differentiation of valvular interstitial cells (VIC). HDAC6 inhibition leads to acetylation of glucose-regulated protein 78 (GRP78), and subsequently induces endoplasmic reticulum $(E R)$ stress. Activating transcription factor 4 (ATF4) activation downstream of ER stress further induces the expression of runt-related transcription factor 2 (Runx2), which promotes the osteoblastic differentiation of VIC. siRNA, Small interfering RNA; ATF6, activating transcription factor 6 . $P E R K$, protein kinase-like ER kinase; $p$-PERK, phosphorylated protein kinase-like ER kinase; IRE1 $\alpha$, inositol-requiring transmembrane kinase and endonuclease- $1 \alpha ; p$-IRE $1 \alpha$, phosphorylated inositol-requiring transmembrane kinase and endonuclease- $1 \alpha$; $p$-eif $2 \alpha$, eukaryotic initiation factor $2 \alpha$; shRNA, short hairpin RNA.

AoV calcification, sob these agents might be cautiously introduced in patients with CAVD and conditions that may lead to CAVD such as uremia. ${ }^{34}$ However, the side effects of HDAC6 inhibitors on AoV calcification need to be further explored in clinical trials.

Our study has some limitations. First, the sample size is small in the human study. We used our data, set alpha as 0.05 and power as 0.8 , to calculate the estimated sample size. The results show that the sample size in the study was enough (immunohistochemical study: control group mean, $28.71 \pm 6.29$; CAVD group mean, $14.07 \pm 2.39$ [lowest $\mathrm{n}=2$ for each group]; Western blot analysis: control group mean, $1.000 \pm 0.404 ; \mathrm{CAVD}$ group mean, $0.393 \pm 0.096$ [lowest $\mathrm{n}=4$ for each group]). However, future studies with a large sample size are needed to confirm the association between HDAC6 levels and the degree of AoV calcification. In addition, the ideal control AoVs should be AoVs from patients who died as a result of head trauma or stroke. The AoVs in our control group may not be the true control because they had moderate aortic insufficiency. Another limitation is that we only used human VICs to explore the mechanisms, although

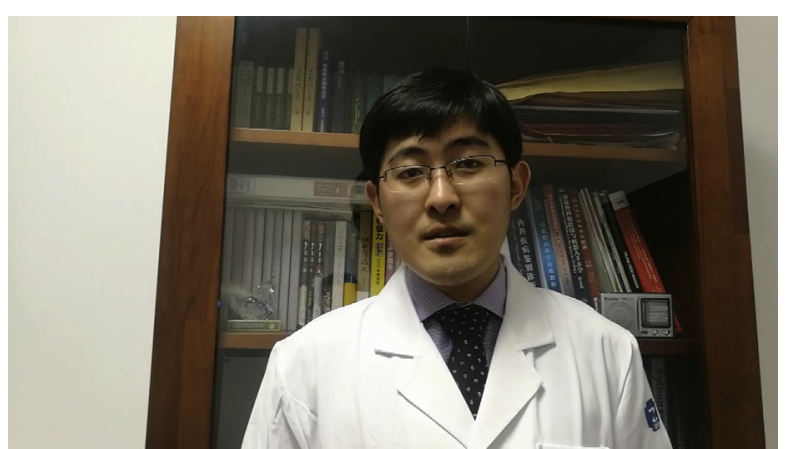

VIDEO 1. The description of the conclusion and significance of the study. Video available at: https://www.jtcvs.org/article/S0022-5223(18)32930-1/ fulltext.

viability and stability of the cells may influence the results. Further in vivo study is required.

\section{CONCLUSIONS}

The present study provides evidence that HDAC6 is associated with AoV calcification. HDAC6 inhibition leads to osteoblast differentiation of VICs via ER stress/ATF4mediated osteogenic pathway. The proposed mechanism is outlined in Figure 7 and Video 1. Our study results indicate that HDAC6 may be a novel target against AoV calcification.

\section{Conflict of Interest Statement}

Authors have nothing to disclose with regard to commercial support.

The authors thank Dr Mingfei Zhao from Roche Tissue Diagnostics for providing statistical assistance.

\section{References}

1. Rajamannan NM, Evans FJ, Aikawa E, Grandeallen KJ, Demer LL, Heistad DD, et al. Calcific aortic valve disease: not simply a degenerative process. Circulation. 2011;124:1783-91.

2. Mathieu P, Boulanger MC. Basic mechanisms of calcific aortic valve disease. Can J Cardiol. 2014;30:982-93.

3. Osnabrugge RL, Mylotte D, Head SJ, Van Mieghem NM, Nkomo VT, Lereun CM, et al. Aortic stenosis in the elderly: disease prevalence and number of candidates for transcatheter aortic valve replacement: a meta-analysis and modeling study. J Am Coll Cardiol. 2013;62:1002-12.

4. Bonow RO, Leon MB, Doshi D, Moat N. Management strategies and future challenges for aortic valve disease. Lancet. 2016;387:1312-23.

5. Pawade TA, Newby DE, Dweck MR. Calcification in aortic stenosis: the skeleton key. J Am Coll Cardiol. 2015;66:561-77.

6. Berndsen CE, Denu JM. Catalysis and substrate selection by histone/protein lysine acetyltransferases. Curr Opin Struct Biol. 2008;18:682-9.

7. Schroeder TM, Westendorf JJ. Histone deacetylase inhibitors promote osteoblast maturation. J Bone Miner Res. 2005;20:2254-63.

8. Azechi T, Kanehira D, Kobayashi T, Sudo R, Nishimura A, Sato F, et al. Trichostatin A, an HDAC class I/II inhibitor, promotes Pi-induced vascular calcification via up-regulation of the expression of alkaline phosphatase. J Atheroscler Thromb. 2013;20:538-47.

9. Kwon DH, Eom GH, Ko JH, Shin S, Joung H, Choe N, et al. MDM2 E3 ligase-mediated ubiquitination and degradation of HDAC1 in vascular calcification. Nat Commun. 2016;7:e201.

10. Cai Z, Li F, Gong W, Liu W, Duan Q, Chen C, et al. Endoplasmic reticulum stress participates in aortic valve calcification in hypercholesterolemic animals. Arterioscler Thromb Vasc Biol. 2013;33:2345-54. 
11. Rao R, Nalluri S, Kolhe R, Yang Y, Fiskus W, Chen J, et al. Treatment with panobinostat induces glucose-regulated protein 78 acetylation and endoplasmic reticulum stress in breast cancer cells. Mol Cancer Ther. 2010;9:942-52.

12. Marciniak SJ, Ron D. Endoplasmic reticulum stress signaling in disease. Physiol Rev. 2006;86:1133-49.

13. Ron D, Walter P. Signal integration in the endoplasmic reticulum unfolded protein response. Nat Rev Mol Cell Biol. 2007;8:519-29.

14. Ni L, Zhou C, Duan Q, Lv J, Fu X, Xia Y, et al. $\beta$-AR Blockers suppresses ER stress in cardiac hypertrophy and heart failure. PLos One. 2011;6:e27294.

15. Myoishi M, Hao H, Minamino T, Watanabe K, Nishihira K, Hatakeyama K, et al. Increased endoplasmic reticulum stress in atherosclerotic plaques associated with acute coronary syndrome. Circulation. 2006;41:1226-33.

16. Wang B, Cai Z, Liu B, Liu Z, Zhou X, Dong N, et al. RAGE deficiency alleviates aortic valve calcification in ApoE-/- mice via the inhibition of endoplasmic reticulum stress. Biochim Biophys Acta Mol Basis Dis. 2017;1863:781-92.

17. Cai Z, Liu B, Wei J, Fu Z, Wang Y, Wang Y, et al. Deficiency of CCAAT/enhancer-binding protein homologous protein (CHOP) prevents diet-induced aortic valve calcification in vivo. Aging Cell. 2017;16:1334-41.

18. Masuda M, Miyazakianzai S, Keenan AL, Shiozaki Y, Okamura K, Chick WS, et al. Activating transcription factor-4 promotes mineralization in vascular smooth muscle cells. JCI Insight. 2016;1:e88646.

19. Abend A, Kehat I. Histone deacetylases as therapeutic targets-from cancer to cardiac disease. Pharmacol Therapeut. 2015;147:55-62.

20. Su SA, Yang D, Wu Y, Xie Y, Zhu W, Cai Z, et al. EphrinB2 regulates cardiac fibrosis through modulating the interaction of Stat 3 and TGF- $\beta /$ Smad 3 signaling. Circ Res. 2017;121:617-27.

21. Fei L, Yao Q, Ao L, Cleveland JC Jr, Dong N, Fullerton DA, et al. Klotho suppresses high phosphate-induced osteogenic responses in human aortic valve interstitial cells through inhibition of Sox 9. J Mol Med. 2017;95:1-13.

22. Yang X, Fullerton DA, Su X, Ao L, Cleveland JC Jr, Meng X. Pro-osteogenic phenotype of human aortic valve interstitial cells is associated with higher levels of Toll-like receptors 2 and 4 and enhanced expression of bone morphogenetic protein 2. J Am Coll Cardiol. 2009;53:491-500.

23. Yoon S, Eom GH. HDAC and HDAC inhibitor: from cancer to cardiovascular diseases. Chonnam Med J. 2016;52:1-11.
24. Zheng XX, Zhou T, Wang XA, Tong XH, Ding JW. Histone deacetylases and atherosclerosis. Atherosclerosis. 2015;240:355-66.

25. Li Z, Zhuang M, Zhang L, Zheng X, Yang P, Li Z. Acetylation modification regulates GRP78 secretion in colon cancer cells. Sci Rep. 2016;6:30406.

26. Yang X, Matsuda K, Bialek P, Jacquot S, Masuoka HC, Schinke T, et al. ATF4 is a substrate of RSK2 and an essential regulator of osteoblast biology; implication for Coffin-Lowry syndrome. Cell. 2004;117:387.

27. Lian N, Lin T, Liu W, Wang W, Li L, Sun S, et al. Transforming growth factor $\beta$ suppresses osteoblast differentiation via the vimentin activating transcription factor 4 (ATF4) axis. J Biol Chem. 2012;287:35975-84.

28. Ducy P, Zhang R, Geoffroy V, Ridall AL, Karsenty G. Osf2/Cbfa1 a transcriptional activator of osteoblast differentiation. Cell. 1997;89:747-54.

29. Lin ME, Chen T, Leaf EM, Speer MY, Giachelli CM. Runx2 expression in smooth muscle cells is required for arterial medial calcification in mice. Am J Pathol. 2015;185:1958-69.

30. Tao H, Yang JJ, Hu W, Shi KH, Li J. HDAC6 Promotes cardiac fibrosis progression through suppressing RASSF1A expression. Cardiology. 2016;133: 18-26.

31. Leng Y, Wu Y, Lei S, Zhou B, Qiu Z, Wang K, et al. Inhibition of HDAC6 activity alleviates myocardial ischemia/reperfusion injury in diabetic rats: potential role of peroxiredoxin 1 acetylation and redox regulation. Oxid Med Cell Longev. 2018;2018:9494052.

32. Kaluza D, Kroll J, Gesierich S, Yao TP, Boon RA, Hergenreider E, et al. Class IIb HDAC6 regulates endothelial cell migration and angiogenesis by deacetylation of cortactin. Embo J. 2011;30:4142-56

33. Yu J, Ma M, Ma Z, Fu J. HDAC6 inhibition prevents TNF- $\alpha$-induced caspase 3 activation in lung endothelial cell and maintains cell-cell junctions. Oncotarget. 2016; 7:54714-22.

34. Kwon DH, Kim YK, Kook H. New aspects of vascular calcification: histone deacetylases and beyond. J Korean Med Sci. 2017;32:1738-48.

Key Words: aortic valve calcification, endoplasmic reticulum stress, histone deacetylase 6 , osteogenesis 


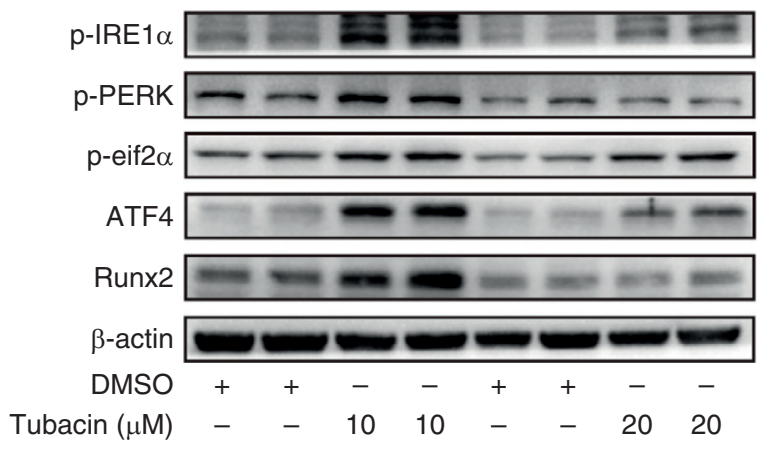

A
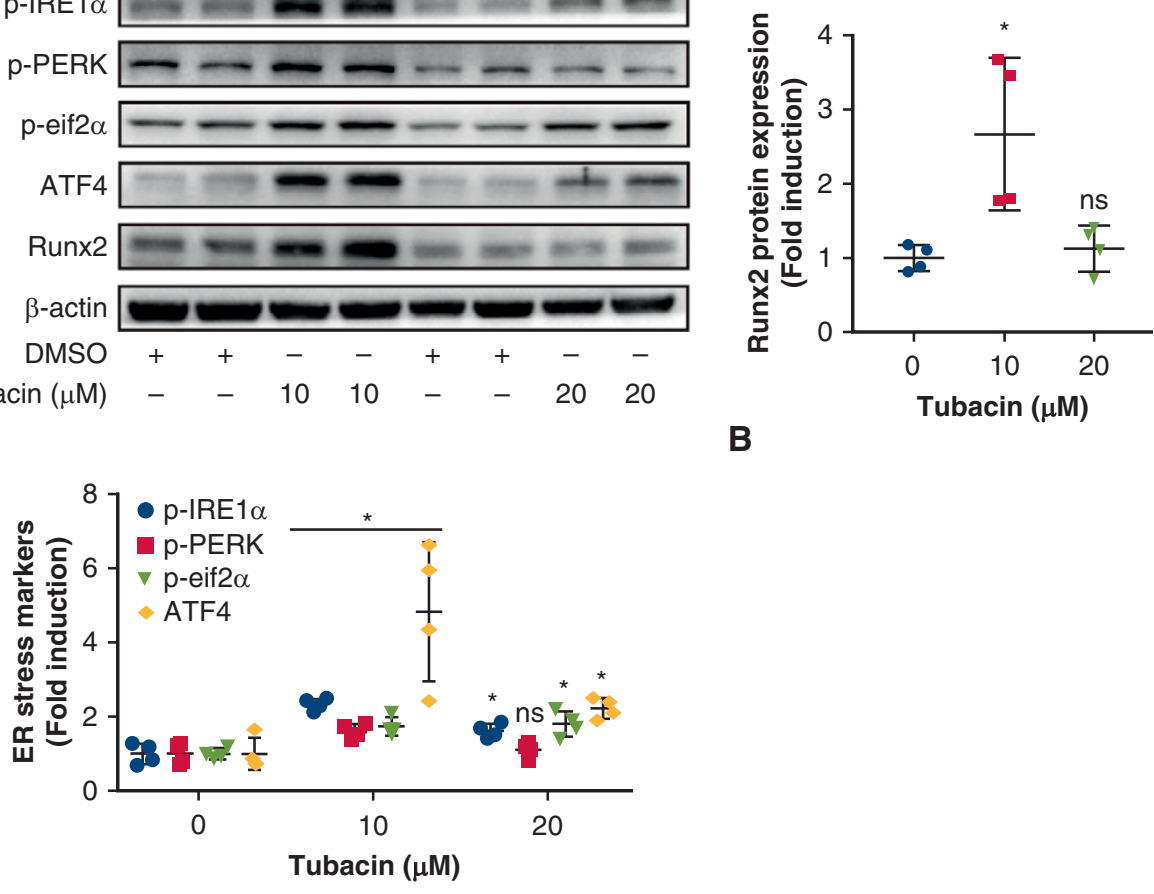

B

C

FIGURE E1. Different concentration of tubacin treatment in valvular interstitial cells (VIC). Treatment with 10 uM tubacin treatment significantly increased expressions of endoplasmic reticulum $(E R)$ stress markers phosphorylated protein kinase-like ER kinase $(p-P E R K)$, phosphorylated inositol-requiring transmembrane kinase and endonuclease- $1 \alpha$ (p-IRE1 $\alpha$ ), phosphorylated eukaryotic initiation factor $2 \alpha$ (p-eif2 $\alpha$ ), activating transcription factor 4 (ATF4), and Runx2 expression in VIC. Treatment with $20 \mathrm{uM}$ tubacin significantly induced p-IRE1 $\alpha$, p-eif $2 \alpha$, and ATF4 expression, but had no significant effect on p-PERK and Runx2 in VIC. A, Western blot of ER stress markers (p-IRE1 $\alpha$, p-PERK, p-eif $2 \alpha$, and ATF4) and Runx 2 after different concentration of tubacin treatment in VIC. B, Statistical analysis of Runx2 protein expression after different concentration of tubacin treatment in VIC. C, Statistical analysis of ER stress markers (p-IRE1 $\alpha$, p-PERK, p-eif $2 \alpha$, and ATF4) after different concentration of tubacin treatment in VIC. $n s$, No significance. $* P<.05$ versus dimethyl sulfoxide (DMSO). Runx2, Runt-related transcription factor 2.
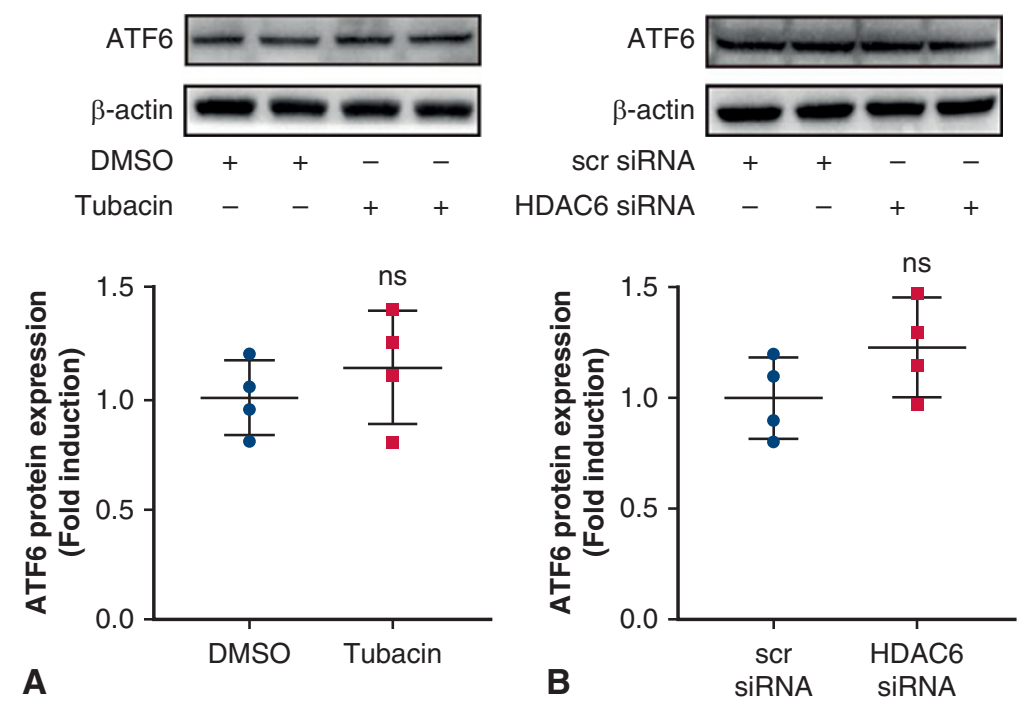

FIGURE E2. Inhibition of histone deacetylase 6 (HDAC6) has no effect on inducing activating transcription factor 6 (ATF6) expression in valvular interstitial cells (VIC). A, Tubacin treatment $(10 \mu \mathrm{M})$ did not induce ATF6 expression in VIC. B, Silencing of HDAC6 did not induce ATF6 expression in VIC. DMSO, Dimethyl sulfoxide; scr siRNA, scrambled small interfering RNA; $n s$, no significance. 

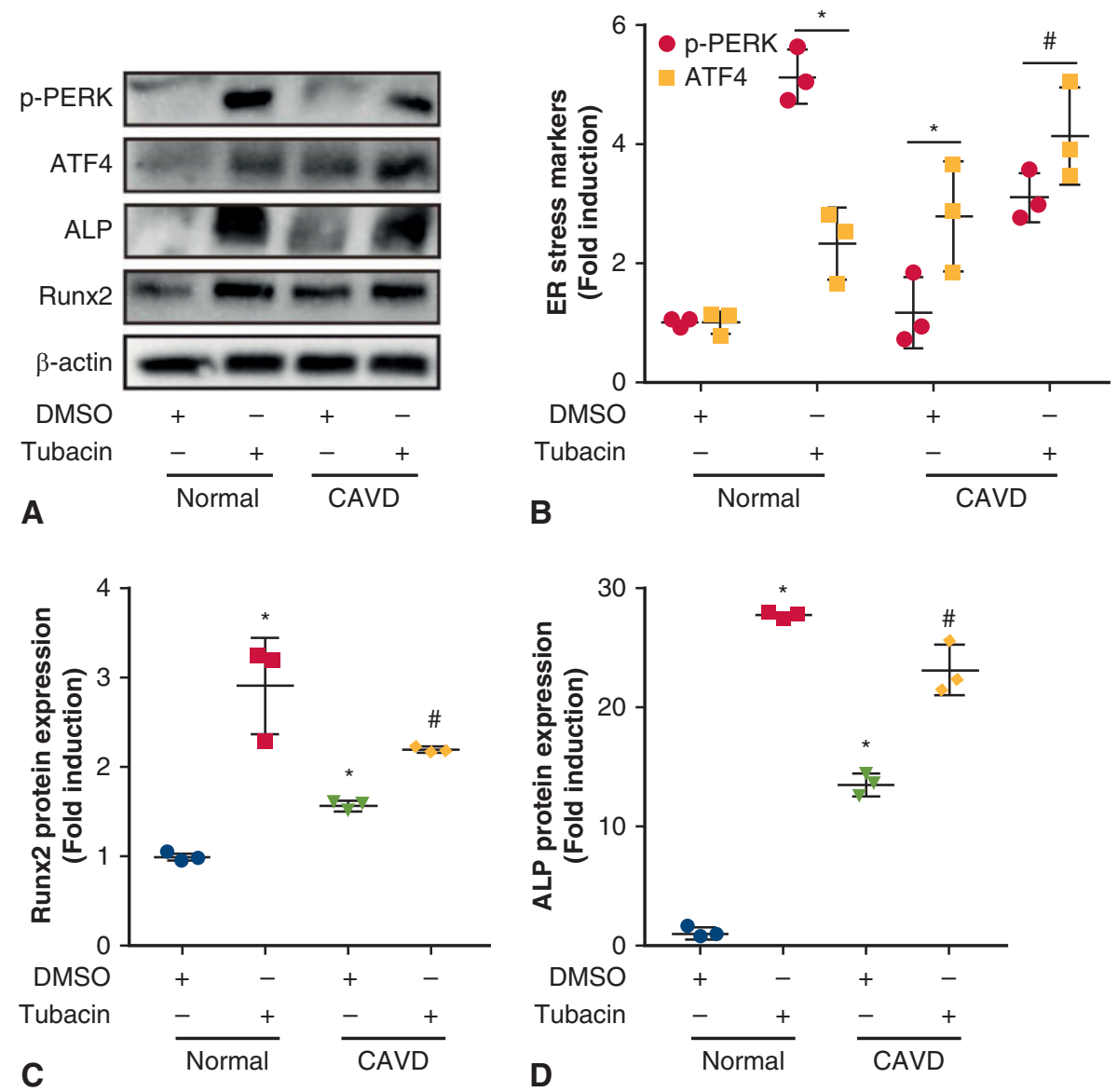

FIGURE E3. The effect of tubacin in valvular interstitial cells (VIC) from patients with calcified aortic valve disease $(C A V D)$ and normal controls. Tubacin treatment $(10 \mu \mathrm{M})$ significantly induced endoplasmic reticulum $(E R)$ stress markers phosphorylated protein kinase-like ER kinase $(p$-PERK) and activating transcription factor 4 (ATF4), as well as Runx2 and alkaline phosphatase (ALP) expression in VIC from calcified aortic valves and in normal controls. ATF4, p-PERK, Runx2, and ALP expressions were significantly increased in VIC from patients with CAVD compared with controls. The effect was more profound in treated VIC from the normal controls. ${ }^{*} P<.05$ versus normal VIC + dimethyl sulfoxide. $\# P<.05$ versus CAVD VIC + dimethyl sulfoxide. Runx2, Runtrelated transcription factor 2; $D M S O$, dimethyl sulfoxide. 